Received: October 2, 2017

Revision received: April 12, 2018

\title{
Study on the influence of exercise on children's cognitive learning ability
}

\author{
Tao Pan \\ Guizhou University of Commerce
}

\begin{abstract}
This paper studies and reveals the relationship between brain development and sport exercise. The degree of brain development has a crucial influence on a person's cognitive learning ability and the development of the entire life trajectory. As we all know, the benefits of exercise are mainly in physical fitness, and the connection between it and the brain science is ignored by common people. By studying the relationship between students' cognitive learning ability and physical exercise, this paper reveals the influence of exercise method and exercise time on the cognitive learning ability such as academic achievement, and observes the effect of exercise behavior on the secretion of white matter cellulose in the brain. Through many examples and brain studies, it's shown that exercise can affect brain structure, enhance brain function, so this paper proposes how to positively and reasonably improve students' attention and academic performance through exercise.
\end{abstract}

\section{Keywords}

Exercise Method $\bullet$ Exercise Time $\bullet$ Cognitive Learning Ability $\bullet$ Brain White Matter Cellulose - Brain Structure 
It is a common aspiration of all the parents in the world to hope their children have a bright future. Therefore, parents are very concerned about their children's academic performance, so it is very important for them to pay attention to their children's cognitive ability. Brain controls people's thoughts and behaviours, from the beginning of life, people have paid special attention to the development of the brain. Childhood is very important for children's character formation and brain development. How to make children form good character and correct cognitive ability in this period is a common concern of the society. Studies have shown that active and proper exercise contributes to children's brain development. Life means movement and physical exercises, and the development and utilization of the brain is inseparable from exercises. People's brains can only exert their potential if their mental and physical strengths are at their best. The researchers found that there are three conditions for brain to be in a good state: the first is to prevent vascular occlusion, the second is to supplement high-quality protein, and the third is to neutralize reactive oxygen. Exercise can exactly meet these conditions of the brain (Barenberg, Berse, \& Dutke, 2011).

At present, scholars at home and abroad mainly use longitudinal experimental intervention studies and horizontal surveys on the study of cognitive ability (Barenberg, Berse, \& Dutke, 2011). In 2010, Travlos conducted a study on students of the eighth-grade, the students were divided into a subject group and a control group. In one day of the school day, 6 PE classes were introduced and arranged at different time periods, and after the physical exercise, students were asked to complete a set of math questions. By the math scores, it proved that active and proper sport exercise can improve the children's mathematics scores (Best, 2012). Hillman, Pontifex, Raine, Castelli \& Hall (2009) divided 20 9-years-old children into two groups, one group took moderate-intensity aerobic exercise and the other group remained seated quietly. The researchers used the flanker task to examine the cognitive control abilities of two groups of children. The results showed that children who had moderate-intensity aerobic exercise had better accuracy in their response, and the effect is even greater under inconsistent conditions. This indicates that children who had physical exercises have better cognitive control ability (Pirrie \& Lodewyk, 2012).

By studying the relationship between learning ability and physical exercise, this paper reveals the influence of exercise time and exercise method on cognitive ability such as academic achievement, and at the same time, it observes the promotion effect of exercise method on the secretion of white matter cellulose in the brain. The age from 6 to 12 is a very important period for the development of children's cognitive ability, and it is also a critical period for the brain development. In daily life and learning, children with good cognitive abilities, whether in terms of academic achievement or personality formation, perform better than children with poor cognitive abilities. They can well control the time to complete the prescribed tasks and can better adapt to unfamiliar environments. This paper illustrates the relationship between students' cognitive ability and physical exercise through typical examples, and proposes how to improve students' academic performance and attention through active and proper exercises.

\section{Influence of exercise type on cognitive learning ability such as academic achievement}

Cognitive function refers to a process in which the human brain processes the received external information 
and converts it into an intrinsic psychology to obtain knowledge or apply knowledge. How to improve cognitive function is very important for the realization of socialization process and the improvement of personal life quality. A large number of studies have shown that exercise can improve cognitive learning functions to a certain extent. However, it should be noted that, it's not that as long as one participates in sports, then one can improve his/her cognitive learning ability, it changes according to different sports situations. Therefore, it's necessary to study what types of exercise can help improve people's cognitive ability. The main elements of the exercise include: intensity, frequency, sport item and duration, etc. (Buck, Hillman, \& Castelli, 2008). Therefore, it is of great theoretical and social practical significance to understand the influence of these exercise factors on human cognitive ability and then adopt certain exercise methods to improve cognitive ability.

According to the classification method of exercise types of the American College of Sports Medicine, exercises can be divided into: aerobic exercise, physical and mental exercise and resistance exercise (Baker et al., 2010). Aerobic exercise refers to physical exercise performed by the human body in the case of adequate oxygen supply; physical and mental exercise refers to a type of method that exerts conscious activity and exercises on its own spirit and form; resistance exercise is a kind of exercise method in which muscles resist resistance and is also a kind of strength exercise.

The experiment method was as follows: randomly selected 100 children aged 8 to 10 who were overweight, they were evenly distributed to the low aerobic exercise group, the high aerobic exercise group, and the control group. Among them, children in the high aerobic exercise group and the low aerobic exercise group participated in the exercise for 20 weeks and 5 days per week, the high aerobic exercise group exercised for 50 minutes every day, and the low aerobic exercise group exercised for 20 minutes every day. The children in the control group did not participate in any exercise. The experimental results are shown in Table 1.

Table 1

Relationship Between High and Low Aerobic Exercise and Cognitive Ability

\begin{tabular}{lcc}
\hline Random control experiment & Exercise time & Effect \\
\hline High aerobic exercise group & 50 minutes & Significant \\
\hline Low aerobic exercise group & 20 minutes & Average \\
\hline Control group & 0 minute & Not particular \\
\hline
\end{tabular}

The results of the study showed that children in the high aerobic exercise group had a significant improvement in their cognitive ability compared to the low aerobic exercise group and the control group. This result is a good proof that high aerobic exercise is beneficial to children's learning and cognitive ability.

Table 2

High and Low Aerobic Exercise Group Performance in Different Tasks

\begin{tabular}{lcc}
\hline & Stroop task & Code recognition task \\
\hline High aerobic exercise group & Good & Good grades \\
\hline Low aerobic exercise group & Average & Poor grades \\
\hline
\end{tabular}

In addition, in the experiment, 100 children aged 8 to 10 who were overweight were randomly selected and divided into a high aerobic exercise group and a low aerobic exercise group. From the aspects of muscle physical strength, body composition and aerobic capacity, the influence of aerobic exercise on children's memory was investigated by the coding recognition task, and the influence of aerobic exercise on learning cognitive ability was investigated by the performance of the Stroop task. The experimental results are shown in Table 2. 
The results of the experiment showed that children in high aerobic exercise group performed better than children in the low aerobic exercise group in the three different Stroop tasks, indicating that high aerobic exercise can make children have better performance in different cognitive function tasks. The experiments of coding recognition tasks show that children's aerobic exercise is an important factor affecting the retrieval, execution and efficient coding of the memory materials.

50 children with an average age of 10 years-old were divided into high and low aerobic exercise groups, and then the flanker task was used to examine the cognitive learning ability of the subjects. The experimental results show that children with high aerobic fitness have a more sensitive response and a higher correct rate. This shows that in the flanker task, there is a certain correlation between children's higher level of aerobic exercise and better performance of the task (Brown et al., 2003).

50 children with an average age of 10 years-old were divided into high and low aerobic exercise groups, and then the fMRI technique was used to examine the block differences between the subjects before and after the flanker task. The experimental results are shown in Table 3.

Table 3

\begin{tabular}{lllll}
\multicolumn{2}{c}{ Different Performances of High and Low Aerobic Exercise Groups in the Block Period } \\
\hline $\begin{array}{l}\text { Frontal area and } \\
\text { parietal area }\end{array}$ & $\begin{array}{l}\text { Early block } \\
\text { period }\end{array}$ & $\begin{array}{l}\text { Late block activation } \\
\text { decline period }\end{array}$ & $\begin{array}{l}\text { Early block } \\
\text { period }\end{array}$ & $\begin{array}{l}\text { Late block activation } \\
\text { decline period }\end{array}$ \\
\hline $\begin{array}{l}\text { High aerobic } \\
\text { exercise group }\end{array}$ & Increase & No change & Increase & Increase \\
\hline $\begin{array}{l}\text { Low aerobic } \\
\text { exercise group }\end{array}$ & Increase & No change & Decrease & Decrease \\
\hline
\end{tabular}

The experimental results show that under the consistent conditions, in the early block period, the recruitment of the frontal and parietal areas of the high and low aerobic exercise groups showed an increase, which was due to the unfamiliarity of the target tasks. Under inconsistent conditions, it can be clearly seen that the subjects in the high aerobic exercise group showed high reaction accuracy regardless of the period. This shows that in order to satisfy and achieve the task, through the control of cognitive ability, the children with high aerobic physical exercise activity have better adaptive neural state in the brain area and can better accomplish the target task.

\section{The influence of time length of exercise on cognitive learning ability such as academic performance}

\section{Study on the influence of short-time exercise on cognitive ability}

Short-time exercise refers to a one-time exercise within a short time. According to the source of energy, it can be divided into anaerobic exercise and aerobic exercise. According to the intensity of exercise, it can be divided into high-intensity exercise, moderate-intensity exercise, and low-intensity exercise (Buck, Hillman \& Castelli, 2008).

In this section, we conducted a study on the influence of short-time moderate-intensity exercise on children's cognitive ability through experiments to reveal the influence of short-time moderate-intensity aerobic exercise 
on children's cognitive ability. From the perspective of brain science, it reveals the mechanism of how shorttime moderate-intensity aerobic exercise influences children's cognitive learning abilities. The experimental results show that about 30-minutes moderate-intensity aerobic exercise can promote children's cognitive ability. It can be seen that short-time moderate-intensity aerobic exercise can stimulate the brain's potential, enhance children's learning ability, and make it easier for children to cultivate good positive learning habits. In addition, in order to study the relationship between short-time exercise and children's cognitive control ability and their academic performance, 30 children with an average age of 10 were randomly selected to complete the cognitive test task in a quiet environment, after the test, these children were assessed for cardiopulmonary fitness. And then, these children were asked to take a 15-minute sprint (the intensity of the exercise was based on $50 \%$ of the maximum oxygen uptake), and after the children's heart rate returned to $15 \%$ of the normal heart rate, they were tested for cognitive ability again. The results of the test showed that children after exercise are more suitable for taking exams than when they are quiet, because they have higher correct rate and better efficiency, which indicates that one-time short-time moderate-intensity aerobic exercise can promote the improvement of children's ability to execute and control, helping them to better accomplish their target tasks.

\section{Study on the influence of long-time exercise on executive function}

Long-time exercise refers to continuous exercise (Chaddock et al., 2010). In 1966, Corder conducted a study on the development of children's intelligence in long-time exercise. Using the WISC test, through a 20-days exercise for children aged between 12-16, the obtained results were not ideal, and there showed no correlation between long-time exercise and intelligence, but whether the 20-days exercise here had reached the effect of long-time exercise requires further researches (Chaddock et al., 2012). In 1967, Brown also did relevant researches, he selected 60 children aged around 12 for a 42-days strength training. The Stanford-Binet test was used, and the experimental results showed that long-time exercise has a significant influence on children's intelligence development (Chaddock, Hillman, Buck, \& Cohen, 2011). In order to study the relationship between long-time exercise and cognitive ability, Catherine L. Davis et al. selected 20 children aged around 7 in 2011 and divided them into a subject group and a control group, and they used MRI to scan their brain activity. The research results indicate that long-time exercise has a positive influence on children's cognitive ability and mathematics performance, bilateral prefrontal cortical activity will increase, bilateral posterior parietal cortical activity will decrease, exercise-induced cognitive ability improvement and brain activation changes have provided experimental evidence and dose-response evidence for the conclusion that exercise can improve brain function in children (Chaddock, Hillman, Buck, \& Cohen, 2011). The results of Catherine L. Davis's findings have provided a reference for education, and this conclusion can convince educators to pay more attention to energetic physical activities.

In conclusion: short-time or long-time moderate-intensity aerobic exercise can improve children's cognitive ability, enhance their control ability, and make them more focused in learning. The experimenters used mice to explore the cause and found that the large consumption of oxygen during exercise caused the blood circulation to accelerate. This oxygen demand increased the number of muscle micro vessels, allowing the muscles to move longer. The same is true for the brain, the faster the blood flows, the more oxygen hemoglobin can be transported to the cells, so you can think more and store more memories. From the results of the research we can see that, 
every student needs to take exercises, on the one hand, maintaining a happy mood makes them read the book well; on the other hand, increasing the blood flow in the brain promotes the expression of the hippocampal neurotrophic factor gene, which helps memory and makes the reading more efficient. Exercise is not a waste of time, it is good for learning.

\section{Effect of exercise behaviour on the secretion of brain white matter celluloses}

In childhood, as the children's brain white matter fasciculus increases, their cognitive learning ability also improves. The main function of the white matter fasciculus in the brain is to connect the gray matter structure of each brain region. The enhanced connectivity between the brain regions means that the brain is constantly maturing, and the generation of reasonable behaviour methods is inseparable from good cooperation between the brain regions. The results of Professor Chaddock-Heyman's research in 2014 showed that the exercise has a secretion-promotion effect on the white matter structure of children's brain, that is, children with higher aerobic exercise fitness have stronger neural connectivity between their brain regions. This change from brain area to brain network has a very important role in improving children's cognitive learning function and test scores. Childhood learning is still mainly in the indoctrination and memory stages, which shows the importance of memory ability to children's learning. Studies have found that, the higher the aerobic exercise fitness, the greater the volume of the bilateral hippocampus, and the higher the memory task scores, and the hippocampus mainly affects the learning memory abilities. Hippocampus is a brain region that helps to deal with long-term learning and memory of sounds and lights, tastes, and other events, and plays the so-called "declarative memory" function. The hippocampus of children's brain is closely linked to aerobic exercises. Short-time moderate-intensity aerobic exercise can promote positive reaction in the hippocampus of the brain, which greatly enhances children's memory (Chaddock et al., 2012). The study found that people with more developed hippocampus have relatively better memories. Children with healthy body around 10 years-old have larger hippocampus than those in unhealthy conditions. This also shows that healthy body is good for improving children's memories (Chang, Nien, Tsai, \& Etnier, 2010).

\section{Conclusion}

By studying the relationship between students' cognitive learning ability and the physical exercise, this paper reveals the influence of exercise method, exercise time on cognitive learning ability such as academic achievement, and observes the effect of exercise behaviour on the secretion of white matter cellulose in the brain. Examples and brain studies have shown that exercise can not only alter the structure of the children's brain, but also affect their brain functions. The childhood brain has good plasticity, and proper and active exercises can help children shape healthy and energetic brains and improve their brain structure and cognitive learning functions. High aerobic and long-time regular physical exercises can help students better absorb knowledge, improve students' cognitive learning ability. Paying attention to the positive promotion of exercise to learning can help the majority of educators to propose more effective learning methods and provide a basis 
for solving real-life teaching problems. In addition, how to use the exercise behaviour to promote the secretion of white matter cellulose in the brain and introduce it into the teaching work is a question that cannot be ignored, and how to expand the hippocampus in children's brain is also very important for the study of children's cognitive ability and learning memory.

\section{References}

Barenberg, J., Berse, T. \& Dutke, S. (2011). Executive functions in learning processes: Do they benefit from physical activity. Educational Research Review, 208, 222. http://dx.doi.org/10.1016/j.edurev.2011.04.002

Best, J.R. (2012). Exergaming Immediately Enhances Children's Executive Function. Developmental Psychology. Advance online publication, 48(5), 1501-1510. http://dx.doi.org/10.1037/a0026648

Pirrie, A.M. \& Lodewyk, K.R. (2012). Investigating links between moderate-to-vigorous physical activity and cognitive performance in elementary school students. Mental Health and Physical Activity, 5(1), 93-98. http://dx.doi.org/10.1016/j.mhpa.2012.04.001

Hillman C.H., Pontifex M.B., Raine L.B., Castelli D.M. \& Hall E.E. (2009), The effect of acute treadmill walking on cognitive control and academic achievement in preadolescent children. Neuroscience, 159(3), 1044-1054. http://dx.doi.org/10.1016/j.neuroscience.2009.01.057

Buck, S.M., Hillman, C. H., \& Castelli, D. M. (2008). The relation of aerobic fitness to stroop task performance in preadolescent children. Medicine and Science in Sports and Exercise, 40(1), 166-172. http://dx.doi.org/10.1249/mss.0b013e318159b035

Baker, L.D., Frank, L.L., Foster-Schubert, K., Green, P.S., Wilkinson, C.W., McTiernan, A. \& Craft, S. (2010). Effects of aerobic exercise on mild cognitive impairment: A controlled trial. Archives of Neurology, 67(1), 71-79. http://dx.doi.org/10.1001/archneurol.2009.307

Brown, J., Cooper-Kuhn, C.M, Kempermann, G., van Praag, H., Winkler, J., Gage, F.H. \& Kuhn, G. (2003). Enriched environment and physical activity stimulate hippocampal but not olfactory bulb neurogenesis. European Journal of Neuroscience, 17(10), 2042-2046. http://dx.doi.org/10.1046/j.14609568.2003.02647.x

Buck, S.M., Hillman, C.H. \& Castelli D.M. (2008). The relation of aerobic fitness to stroop task performance in preadolescent children. Medicine and Science in Sports and Exercise, 40(1), 166-172. http://dx.doi.org/10.1249/mss.0b013e318159b035

Chaddock, L., Erickson, K.I., Prakash, R.S., VanPatter, M., Voss, M.W., Pontifex, M.B. \& Kramer, A.F. (2010). Basal ganglia volume is associated with aerobic fitness in preadolescent children. Developmental Neuroscience, 32(3), 249-256. http://dx.doi.org/10.1159/000316648

Chaddock, L., Erickson, K.I., Prakash, R.S., Voss, M.W., VanPatter, M., Pontifex, M.B. \& Kramer, A.F. (2012). A functional MRI investigation of the association between childhood aerobic fitness and neurocognitive control. Biological Psychology, 89(1), 260-268. http://dx.doi.org/10.1016/j.biopsycho.2011.10.017

Chaddock, L., Hillman, C.H., Buck, S.M. \& Cohen, N.J. (2011). Aerobic fitness and executive control of relational memory in preadolescent children. Medicine and Science in Sports and Exercise, 43(2), 344-349. http://dx.doi.org/10.1249/MSS.0b013e3181e9af48 
Chaddock, L., Hillman, C.H., Pontifex, M.B., Johnson, C.R., Raine, L.B., \& Kramer, A.F. (2012). Childhood aerobic fitness predicts cognitive performance one year later. Journal of Sports Sciences, 30(5), 421-430. http://dx.doi.org/10.1080/02640414.2011.647706

Chang, Y.K., Nien, Y.H., Tsai, C.L., \& Etnier, J.L. (2010). Physical activity and cognition in older adults: The potential of Tai Chi Chuan. Journal of Aging and Physical Activity, 18(4), 451-472. http://dx.doi.org/10.1123/japa.18.4.451 